\title{
ACCURATE RECONSTRUCTIONS OF FUNCTIONS OF FINITE REGULARITY FROM TRUNCATED FOURIER SERIES EXPANSIONS
}

\author{
KNUT S. ECKHOFF
}

\begin{abstract}
Knowledge of a truncated Fourier series expansion for a $2 \pi$ periodic function of finite regularity, which is assumed to be piecewise smooth in each period, is used to accurately reconstruct the corresponding function. An algebraic equation of degree $M$ is constructed for the $M$ singularity locations in each period for the function in question. The $M$ coefficients in this algebraic equation are obtained by solving an algebraic system of $M$ equations determined by the coefficients in the known truncated expansion. If discontinuities in the derivatives of the function are considered, in addition to discontinuities in the function itself, that algebraic system will be nonlinear with respect to the $M$ unknown coefficients. The degree of the algebraic system will depend on the desired order of accuracy for the reconstruction, i.e., a higher degree will normally lead to a more accurate determination of the singularity locations. By solving an additional linear algebraic system for the jumps of the function and its derivatives up to the arbitrarily specified order at the calculated singularity locations, we are able to reconstruct the $2 \pi$-periodic function of finite regularity as the sum of a piecewise polynomial function and a function which is continuously differentiable up to the specified order.
\end{abstract}

\section{INTRODUCTION}

With a Riemann integrable complex-valued $2 \pi$-periodic function $u(x)$ we may associate, for any given even integer $N>0$, the $N$ th-order truncated Fourier series

$$
P_{N} u(x)=\sum_{k=-N / 2+1}^{N / 2-1} \hat{u}_{k} e^{i k x}
$$

Received by the editor March 16, 1992 and, in revised form, July 12, 1993 and January 26, 1994.

1991 Mathematics Subject Classification. Primary 65M70, 65N35.

This paper is partly based on work done while the author was engaged at the SINTEF Multiphase Flow Laboratory, Trondheim, Norway. The research was supported by NTNF Contract No. OT45.23626 under the Program for multiphase flow technology (PROFF) 1989-91. The support from The Royal Norwegian Council for Scientific and Industrial Research (NTNF), Amoco, Elf, Esso, Norsk Hydro, Saga, Shell, Statoil, Total, Aker Engineering, Framo Engineering, Norwegian Applied Technology, Kværner Engineering, and SINTEF is thankfully acknowledged. 
where

$$
\hat{u}_{k}=\frac{1}{2 \pi} \int_{-\pi}^{\pi} u(x) e^{-i k x} d x, \quad k=0, \pm 1, \pm 2, \ldots
$$

As is well known $[1,8,16]$, the error involved, when we approximate $u(x)$ by the truncated Fourier series expansion (1), is strongly dependent on the smoothness of the function $u(x)$. We shall in this paper limit our discussion to functions $u(x)$ which are piecewise smooth on $[-\pi, \pi]$. If in addition we assume that $u(x)$ is everywhere continuous and has continuous derivatives of order $p=1,2, \ldots, m-1$, it can be shown $[1,8,16]$ that

$$
\hat{u}_{k}=O\left(|k|^{-(m+1)}\right) \text { as } k \rightarrow \pm \infty .
$$

For a discontinuous function $u(x)$, the relation (3) holds with $m=0$, and consequently convergence of the associated Fourier series is very slow. The resulting oscillatory behavior of the truncated Fourier series expansion (1) near a discontinuity, known as the Gibbs phenomenon, is usually regarded as the major cause of the problems connected with the application of Fourier methods (as well as other types of spectral methods) to the calculation of shock waves. As formulated recently by Gottlieb [6], for instance, one fundamental problem in this connection is to design a method which makes it possible to recover, with high accuracy, pointwise values of a piecewise smooth function from its first $N$ Fourier coefficients. A solution to that problem is the purpose of the present paper.

The oscillatory behavior obtained by a high-order method like a spectral method should according to Lax [10] contain enough information for us to be able to reconstruct the proper nonoscillatory discontinuous function by a postprocessing filter. The idea of introducing step functions for that purpose, initiated by Gottlieb et al. [7] and further elaborated on in [3], will in this paper be extended to $2 \pi$-periodic functions of finite regularity that involve discontinuities in the derivatives up to an arbitrarily specified order, in addition to discontinuities in the function itself. The resulting reconstruction algorithms will be of a similar nature as those described in [3], but will lead to more accurate reconstructions at the expense of more involved calculations. We note that ideas similar to some in this paper have been exploited earlier for various other problems related to Fourier analysis [11, 12].

\section{THE EXTENDED PROBLEM OF RECONSTRUCTION}

In the reconstruction of discontinuous $2 \pi$-periodic functions described in [3] we utilized a one-parameter family of $2 \pi$-periodic step functions (or rather sawtooth functions), which on the interval $(-\pi, \pi]$ may be given by

$$
V_{0}(x ; \beta)= \begin{cases}\frac{1}{2 \pi}(\beta-\pi-x) & \text { if }-\pi<x<\beta, \\ \frac{1}{2 \pi}(\beta+\pi-x) & \text { if } \beta<x \leq \pi,\end{cases}
$$


where $\beta$ is a parameter at our disposal, with $-\pi \leq \beta<\pi$. The function (4) has a jump discontinuity of magnitude +1 at $x=\beta .^{1}$ Hence $V_{0}(x ; \beta)$ is a piecewise linear $2 \pi$-periodic function with exactly one discontinuity in each period and no jump in the derivatives at the points of discontinuity. Starting with this function, we may successively define a sequence of functions $V_{n}(x ; \beta)$ by

$$
V_{n}(x ; \beta)=\int V_{n-1}(x ; \beta) d x, \quad n=1,2, \ldots,
$$

where the constants of integration are determined by

$$
\int_{-\pi}^{\pi} V_{n}(x ; \beta) d x=0, \quad n=1,2, \ldots
$$

From this definition it follows that for each $n=1,2, \ldots, V_{n}(x ; \beta)$ is a $2 \pi$-periodic function of finite regularity with $V_{n}^{(p)}(x ; \beta)$ continuous everywhere for $p=0, \ldots, n-1$, but with $V_{n}^{(n)}(x ; \beta)=V_{0}(x ; \beta)$ only piecewise continuous with jump discontinuities of magnitude +1 at $x=\beta+2 m \pi$, $m=0, \pm 1, \pm 2, \ldots$. For the higher-order derivatives $V_{n}^{(p)}(x ; \beta), p \geq n+1$, there are no jumps at these singularity locations.

More specifically, it follows from (4), (5), and (6) that $V_{n}(x ; \beta)=U_{n}(x-\beta)$, where $U_{n}(\xi)$ for $n=0,1,2, \ldots$ is the $2 \pi$-periodic extension of the following function:

$$
U_{n}(\xi)=-\frac{(2 \pi)^{n}}{(n+1) !} B_{n+1}\left(\frac{\xi}{2 \pi}\right) \text { when } 0<\xi<2 \pi,
$$

where $B_{j}(x), j=1,2, \ldots$, are the Bernoulli polynomials [4]. We note that $U_{n}(\xi)$ is an odd function when $n$ is even, and an even function when $n$ is odd.

From (4), (5), and (6) it is not difficult to see that the Fourier coefficients (2) for the functions $V_{n}(x ; \beta), n=0,1,2, \ldots$, are given by

$$
\left.\widehat{(V}_{n}\right)_{0}(\beta)=0, \quad \widehat{(V)}_{n}(\beta)=\frac{e^{-i k \beta}}{2 \pi(i k)^{n+1}}, \quad k= \pm 1, \pm 2, \ldots
$$

If we now consider a $2 \pi$-periodic function $u(x)$ which is known to be piecewise smooth on $[-\pi, \pi]$, the interval $[-\pi, \pi]$ can be divided into a finite number of subintervals on which $u(x)$ is smooth. At the endpoints of these subintervals, however, the function $u(x)$ and/or some (or all) of its derivatives may have jump discontinuities. Thus, the assumption that the $2 \pi$-periodic function of finite regularity $u(x)$ is piecewise smooth on $[-\pi, \pi]$ is clearly equivalent to the assumption that $u(x)$, for an arbitrarily given nonnegative integer $Q$, can be written in the following way for some finite integer $M \geq 1$ :

$$
u(x)=v(x)+\sum_{n=0}^{Q} \sum_{j=1}^{M} A_{j}^{n} V_{n}\left(x ; \gamma_{j}\right),
$$

where $v(x)$ is some $Q$ times continuously differentiable $2 \pi$-periodic function which is piecewise smooth on $[-\pi, \pi]$, the functions $V_{n}\left(x ; \gamma_{j}\right)$ are given by

\footnotetext{
${ }^{1}$ In this paper we apply the convention that function values are disregarded at points where a piecewise smooth function is discontinuous.
} 
(4), (5), (6) with $\beta=\gamma_{j}$, and $A_{j}^{n}, \gamma_{j}$ are some constants for $j=1, \ldots, M$, $n=0,1, \ldots, Q$. From the properties of the functions $V_{n}(x ; \beta)$ discussed above, it is clear that the points $x=\gamma_{j}, j=1, \ldots, M$, are the locations for the singularities of the function $u(x)$ given by (9), and $A_{j}^{0}$ are the associated jumps for the function $u(x)$ itself, $A_{j}^{1}$ those for $u^{\prime}(x), A_{j}^{2}$ those for $u^{\prime \prime}(x)$, and so on, until finally $A_{j}^{Q}$ are the associated jumps for $u^{(Q)}(x)$ at the singularity locations.

Clearly, there will be no loss of generality by assuming that $-\pi \leq \gamma_{1}<$ $\gamma_{2}<\ldots<\gamma_{M}<\pi$. The sum on the right-hand side in (9) is seen to constitute a $2 \pi$-periodic piecewise polynomial function of degree at most $Q+1$, i.e., it is a polynomial of degree at most $Q+1$ on each of the intervals $\left(\gamma_{M}-2 \pi, \gamma_{1}\right),\left(\gamma_{1}, \gamma_{2}\right),\left(\gamma_{2}, \gamma_{3}\right), \ldots,\left(\gamma_{M-1}, \gamma_{M}\right),\left(\gamma_{M}, \gamma_{1}+2 \pi\right)$, and so on. The problem we study in this paper is that of determining good approximations for the function $v(x)$ and the constants $A_{j}^{n}, \gamma_{j}$ in (9) solely from knowledge of a finite number of Fourier coefficients associated with the function $u(x)$. For the case where $Q=0$, this is exactly the problem considered in [3]. In the following we shall therefore assume that $Q \geq 1$, and we furthermore assume that the integer $M$ is known. As we shall briefly discuss in $\S 6$, it is also possible (by essentially the same method) to handle cases where the integer $M$ is not known a priori, and in fact to determine the integer $M$ as part of the reconstruction.

The Fourier coefficients (2) associated with the function $u(x)$ given by (9) are found from (8) to be

$$
\hat{u}_{k}=\hat{v}_{k}+\sum_{n=0}^{Q} \frac{1}{2 \pi(i k)^{n+1}} \sum_{j=1}^{M} A_{j}^{n} e^{-i k \gamma_{j}}, \quad k= \pm 1, \pm 2, \ldots,
$$

and $\hat{u}_{0}=\hat{v}_{0}$. With the notation

$$
C_{k}=2 \pi i k\left(\hat{u}_{k}-\hat{v}_{k}\right), \quad k= \pm 1, \pm 2, \ldots,
$$

the relations (10) can be written

$$
\sum_{n=0}^{Q} \frac{1}{(i k)^{n}} \sum_{j=1}^{M} A_{j}^{n} e^{-i k \gamma_{j}}=C_{k}, \quad k= \pm 1, \pm 2, \ldots
$$

Since the function $v(x)$ in (9) is assumed to be $Q$ times continuously differentiable everywhere and piecewise smooth on $[-\pi, \pi]$, we have by $(3)$

$$
\hat{v}_{k}=O\left(|k|^{-Q-2}\right) \text { as } k \rightarrow \pm \infty .
$$

On the other hand, if we restrict our study to cases where $u(x)$ is a discontinuous function, we get by (3)

$$
\hat{u}_{k}=O\left(|k|^{-1}\right) \text { as } k \rightarrow \pm \infty,
$$

where the order relation in (14) is best possible [16]. As for the case $Q=0$ discussed in [3], it is this difference in behavior of the Fourier coefficients, (14) vs. (13), which forms the basis for our reconstruction procedure.

For $|k|$ sufficiently large, in view of (11), (13), (14) we can expect that

$$
C_{k} \approx 2 \pi i k \hat{u}_{k} \stackrel{\text { def }}{=} \tilde{C}_{k}=O(1) \text { as } k \rightarrow \pm \infty,
$$


since the difference between the two sides in (15) is asymptotically of the order $O\left(|k|^{-Q-1}\right)$ as $k \rightarrow \pm \infty$. Introduced into (12), the approximation (15) gives us approximate equations for the quantities $A_{j}^{n}$ and $\gamma_{j}$. The hardest part of the reconstruction is the determination of the singularity locations $\gamma_{j}, j=$ $1, \ldots, M$, with sufficient accuracy from these equations. We shall therefore postpone that problem and for the moment assume that the singularity locations $\gamma_{j}, j=1, \ldots, M$, are known to an accuracy of the order $O\left(|N|^{-Q-2}\right)$ as $N \rightarrow$ $\infty$. From (12) and (15) we then get the following linear system of approximate algebraic equations for the amplitudes $A_{j}^{n}, j=1, \ldots, M, n=0,1, \ldots, Q$ :

$$
\sum_{n=0}^{Q} \frac{1}{(i k)^{n}} \sum_{j=1}^{M} A_{j}^{n} e^{-i k \gamma_{j}}=\tilde{C}_{k},
$$

for $k=N / 2-M(Q+1), N / 2-M(Q+1)+1, \ldots, N / 2-1$. As partially discussed in [3], we have here chosen $M(Q+1)$ consecutive values of $k$ in order to ensure that the equations in (16) are linearly independent. Clearly, this implies that we normally must have $N$ larger than $2 M(Q+1)$, and with the assumptions made we can expect that for each $j=1, \ldots, M$ the error in the approximately determined quantity $A_{j}^{n}$ is of the order $O\left(|N|^{-Q-1+n}\right)$ as $N \rightarrow \infty$ for $n=0,1, \ldots, Q$.

When the amplitudes $A_{j}^{n}$ have been determined by (16), an approximate function $v(x)$ in (9) can be determined by an $N$ th-order truncated Fourier series (1) with Fourier coefficients $\hat{v}_{k}$ given by

$$
\hat{v}_{k}=\hat{u}_{k}-\sum_{n=0}^{Q} \frac{1}{2 \pi(i k)^{n+1}} \sum_{j=1}^{M} A_{j}^{n} e^{-i k \gamma_{j}},
$$

for $k= \pm 1, \pm 2, \ldots, \pm(N / 2-1)$, and $\hat{v}_{0}=\hat{u}_{0}$.

It remains to determine the singularity locations $\gamma_{j}, j=1, \ldots, M$, with sufficient accuracy. As we shall see in the following sections, this is possible to do from (12), (15) if these equations are considered for a sufficient number of sufficiently large values of $|k|$. As in [3], the key to the solution of that problem is to construct a sufficiently accurate approximation to the algebraic equation of degree $M$,

$$
z^{M}+X_{1} z^{M-1}+X_{2} z^{M-2}+\cdots+X_{M-1} z+X_{M}=0
$$

with roots $z_{j}=e^{-i \gamma_{j}}, j=1, \ldots, M$. In order to facilitate the bookkeeping, we introduce for $n=0,1,2, \ldots, Q$, the notation

$$
B_{k}^{n} \stackrel{\text { def }}{=} \sum_{j=1}^{M} A_{j}^{n} e^{-i k \gamma_{j}}, \quad k= \pm 1, \pm 2, \ldots
$$

In essentially the same way as discussed in [3], we then find as a consequence of Prony's method [15] (as presented in [9]) that the coefficients $X_{j}, j=$ $1, \ldots, M$, in (18) must satisfy the identities

$$
B_{k}^{n}+B_{k-1}^{n} X_{1}+B_{k-2}^{n} X_{2}+\cdots+B_{k-M}^{n} X_{M}=0,
$$

for each $n=0,1,2, \ldots, Q$, and for every $k \neq 0,1,2, \ldots, M$. 
Unfortunately, however, none of the quantities $B_{j}^{n}$ are a priori known from the Fourier coefficients $\hat{u}_{k}, k=0, \pm 1, \pm 2, \ldots, \pm(N / 2-1)$, with sufficient accuracy when $Q \geq 1$. From (12) and (19) we do know, however, that the combinations

$$
\sum_{n=0}^{Q} \frac{1}{(i k)^{n}} B_{k}^{n}=C_{k}, \quad k= \pm 1, \pm 2, \ldots,
$$

are approximately given by (15). In the following sections we shall describe how this can be utilized in an economical way to determine approximations for the coefficients $X_{j}$ in (18) such that the $M$ singularity locations $\gamma_{j}, j=$ $1, \ldots, M$, can be determined with sufficient accuracy.

\section{THE LOCATION OF THE SINGULARITIES}

3.1. The case $Q=1$. In the present case, the relations (21) can be written

$$
i k B_{k}^{0}+B_{k}^{1}=i k C_{k}, \quad k= \pm 1, \pm 2, \ldots
$$

From the identities (20) and (22) we get for every $k \neq 0,1,2, \ldots, M$

$$
\begin{aligned}
D_{k} \stackrel{\text { def }}{=} & k C_{k}+(k-1) C_{k-1} X_{1}+(k-2) C_{k-2} X_{2}+\cdots+(k-M) C_{k-M} X_{M} \\
= & k B_{k}^{0}+(k-1) B_{k-1}^{0} X_{1}+(k-2) B_{k-2}^{0} X_{2}+\cdots+(k-M) B_{k-M}^{0} X_{M} \\
& -i\left\{B_{k}^{1}+B_{k-1}^{1} X_{1}+B_{k-2}^{1} X_{2}+\cdots+B_{k-M}^{1} X_{M}\right\} \\
= & -B_{k-1}^{0} X_{1}-2 B_{k-2}^{0} X_{2}-\cdots-M B_{k-M}^{0} X_{M} .
\end{aligned}
$$

Furthermore, it follows from (20) and (23) that for every $k \neq 0,1,2, \ldots, 2 M$ (24)

$$
\begin{aligned}
D_{k}+ & D_{k-1} X_{1}+D_{k-2} X_{2}+\cdots+D_{k-M} X_{M} \\
= & -X_{1}\left\{B_{k-1}^{0}+B_{k-2}^{0} X_{1}+B_{k-3}^{0} X_{2}+\cdots+B_{k-M-1}^{0} X_{M}\right\} \\
& -2 X_{2}\left\{B_{k-2}^{0}+B_{k-3}^{0} X_{1}+B_{k-4}^{0} X_{2}+\cdots+B_{k-M-2}^{0} X_{M}\right\} \\
& -\cdots-M X_{M}\left\{B_{k-M}^{0}+B_{k-M-1}^{0} X_{1}+B_{k-M-2}^{0} X_{2}+\cdots+B_{k-2 M}^{0} X_{M}\right\}=0 .
\end{aligned}
$$

From (15) and (23) it is clear that the quantities $D_{k}$ are approximately known from the Fourier coefficients associated with $u(x)$ in the following way:

$$
\begin{aligned}
D_{k} \approx \tilde{D}_{k} \stackrel{\text { def }}{=} & k \tilde{C}_{k}+(k-1) \tilde{C}_{k-1} X_{1}+(k-2) \tilde{C}_{k-2} X_{2} \\
& +\cdots+(k-M) \tilde{C}_{k-M} X_{M}=O(k) \text { as } k \rightarrow \pm \infty .
\end{aligned}
$$

The difference between the two sides in (25) is of the order $O\left(|k|^{-1}\right)$ as $k \rightarrow$ $\pm \infty$. An approximate system of equations for the coefficients $X_{j}, j=1, \ldots$, $M$, in (18), and hence for the singularity locations for the function $u(x)$, results from (24) and (25):

$$
\tilde{D}_{k}+\tilde{D}_{k-1} X_{1}+\tilde{D}_{k-2} X_{2}+\cdots+\tilde{D}_{k-M} X_{M}=0,
$$

where we normally take $k=N / 2-M, \ldots, N / 2-1$, assuming that $N>$ $6 M$. This choice is made in order to ensure that the equations in (26) are 
independent. From (25) it is clear that (26) is an algebraic equation of degree 2 for the coefficients $X_{j}, j=1, \ldots, M$. We shall in later sections discuss how the equations (26) can be solved. At this stage we only observe that from the estimates we have given above, we can expect that the error in the solution of (26) for $X_{j}$ will be of the order $O\left(|N|^{-2}\right)$ as $N \rightarrow \infty$. As we shall discuss in more detail in $\S 4$, this is one order better than the error we can expect in general for the method described in [3] when $Q=0$.

3.2. The case $Q=2$. In this case, the relations (21) can be written

$$
(i k)^{2} B_{k}^{0}+i k B_{k}^{1}+B_{k}^{2}=(i k)^{2} C_{k}, \quad k= \pm 1, \pm 2, \ldots
$$

From the identities (20) and (27) we get for every $k \neq 0,1,2, \ldots, M$

$$
\begin{aligned}
E_{k} \stackrel{\text { def }}{=} & k^{2} C_{k}+(k-1)^{2} C_{k-1} X_{1}+(k-2)^{2} C_{k-2} X_{2}+\cdots+(k-M)^{2} C_{k-M} X_{M} \\
= & k^{2} B_{k}^{0}+(k-1)^{2} B_{k-1}^{0} X_{1}+(k-2)^{2} B_{k-2}^{0} X_{2}+\cdots+(k-M)^{2} B_{k-M}^{0} X_{M} \\
& -i k B_{k}^{1}-i(k-1) B_{k-1}^{1} X_{1}-i(k-2) B_{k-2}^{1} X_{2}-\cdots-i(k-M) B_{k-M}^{1} X_{M} \\
& -B_{k}^{2}-B_{k-1}^{2} X_{1}-B_{k-2}^{2} X_{2}-\cdots-B_{k-M}^{2} X_{M} \\
= & -2 k B_{k-1}^{0} X_{1}-4 k B_{k-2}^{0} X_{2}-\cdots-2 M k B_{k-M}^{0} X_{M} \\
& +B_{k-1}^{0} X_{1}+4 B_{k-2}^{0} X_{2}+\cdots+M^{2} B_{k-M}^{0} X_{M} \\
& +i B_{k-1}^{1} X_{1}+2 i B_{k-2}^{1} X_{2}+\cdots+M i B_{k-M}^{1} X_{M} .
\end{aligned}
$$

From (28) it follows that for every $k \neq 0,1,2, \ldots, 2 M$

$$
\begin{aligned}
F_{k} \stackrel{\text { def }}{=} & E_{k}+E_{k-1} X_{1}+E_{k-2} X_{2}+\cdots+E_{k-M} X_{M} \\
= & -2 X_{1}\left\{k B_{k-1}^{0}+(k-1) B_{k-2}^{0} X_{1}+\cdots+(k-M) B_{k-M-1}^{0} X_{M}\right\} \\
& -4 X_{2}\left\{k B_{k-2}^{0}+(k-1) B_{k-3}^{0} X_{1}+\cdots+(k-M) B_{k-M-2}^{0} X_{M}\right\} \\
& -\cdots-2 M X_{M}\left\{k B_{k-M}^{0}+(k-1) B_{k-M-1}^{0} X_{1}+\cdots+(k-M) B_{k-2 M}^{0} X_{M}\right\} \\
& +X_{1}\left\{B_{k-1}^{0}+B_{k-2}^{0} X_{1}+B_{k-3}^{0} X_{2}+\cdots+B_{k-M-1}^{0} X_{M}\right\} \\
& +4 X_{2}\left\{B_{k-2}^{0}+B_{k-3}^{0} X_{1}+B_{k-4}^{0} X_{2}+\cdots+B_{k-M-2}^{0} X_{M}\right\} \\
& +\cdots+M^{2} X_{M}\left\{B_{k-M}^{0}+B_{k-M-1}^{0} X_{1}+B_{k-M-2}^{0} X_{2}+\cdots+B_{k-2 M}^{0} X_{M}\right\} \\
& +i X_{1}\left\{B_{k-1}^{1}+B_{k-2}^{1} X_{1}+B_{k-3}^{1} X_{2}+\cdots+B_{k-M-1}^{1} X_{M}\right\} \\
& +2 i X_{2}\left\{B_{k-2}^{1}+B_{k-3}^{1} X_{1}+B_{k-4}^{1} X_{2}+\cdots+B_{k-M-2}^{1} X_{M}\right\} \\
& +\cdots+M i X_{M}\left\{B_{k-M}^{1}+B_{k-M-1}^{1} X_{1}+B_{k-M-2}^{1} X_{2}+\cdots+B_{k-2 M}^{1} X_{M}\right\} .
\end{aligned}
$$

Again, by (20), it follows from (29) that

$$
\begin{aligned}
F_{k}= & 2 X_{1}\left\{B_{k-2}^{0} X_{1}+2 B_{k-3}^{0} X_{2}+\cdots+M B_{k-M-1}^{0} X_{M}\right\} \\
& +4 X_{2}\left\{B_{k-3}^{0} X_{1}+2 B_{k-4}^{0} X_{2}+\cdots+M B_{k-M-2}^{0} X_{M}\right\} \\
& +\cdots+2 M X_{M}\left\{B_{k-M-1}^{0} X_{1}+2 B_{k-M-2}^{0} X_{2}+\cdots+M B_{k-2 M}^{0} X_{M}\right\}
\end{aligned}
$$


Finally, from (20) and (30) it follows that for every $k \neq 0,1,2, \ldots, 3 M$

$$
\begin{aligned}
F_{k}+ & F_{k-1} X_{1}+F_{k-2} X_{2}+\cdots+F_{k-M} X_{M} \\
= & 2 X_{1} X_{1}\left\{B_{k-2}^{0}+B_{k-3}^{0} X_{1}+\cdots+B_{k-M-2}^{0} X_{M}\right\} \\
& +4 X_{1} X_{2}\left\{B_{k-3}^{0}+B_{k-4}^{0} X_{1}+\cdots+B_{k-M-3}^{0} X_{M}\right\} \\
& +\cdots+2 M X_{1} X_{M}\left\{B_{k-M-1}^{0}+B_{k-M-2}^{0} X_{1}+\cdots+B_{k-2 M-1}^{0} X_{M}\right\} \\
& +4 X_{2} X_{1}\left\{B_{k-3}^{0}+B_{k-4}^{0} X_{1}+\cdots+B_{k-M-3}^{0} X_{M}\right\} \\
& +\cdots+4 M X_{2} X_{M}\left\{B_{k-M-2}^{0}+B_{k-M-3}^{0} X_{1}+\cdots+B_{k-2 M-2}^{0} X_{M}\right\} \\
& +\cdots+2 M X_{M} X_{1}\left\{B_{k-M-1}^{0}+B_{k-M-2}^{0} X_{1}+\cdots+B_{k-2 M-1}^{0} X_{M}\right\} \\
& +\cdots+2 M^{2} X_{M} X_{M}\left\{B_{k-2 M}^{0}+B_{k-2 M-1}^{0} X_{1}+\cdots+B_{k-3 M}^{0} X_{M}\right\}=0 .
\end{aligned}
$$

From (15) and (28) it is clear that the quantities $E_{k}$ are approximately expressible in terms of the Fourier coefficients of $u(x)$ :

$$
\begin{gathered}
E_{k} \approx \tilde{E}_{k} \stackrel{\text { def }}{=} k^{2} \tilde{C}_{k}+(k-1)^{2} \tilde{C}_{k-1} X_{1}+(k-2)^{2} \tilde{C}_{k-2} X_{2} \\
+\cdots+(k-M)^{2} \tilde{C}_{k-M} X_{M}=O\left(k^{2}\right) \text { as } k \rightarrow \pm \infty
\end{gathered}
$$

The difference between the two sides in (32) is seen to be of the order $O\left(|k|^{-1}\right)$ as $k \rightarrow \pm \infty$. With the same accuracy, we obtain from (29) and (32) the following approximations:

$$
\begin{aligned}
F_{k} & \approx \tilde{F}_{k} \stackrel{\text { def }}{=} \tilde{E}_{k}+\tilde{E}_{k-1} X_{1}+\tilde{E}_{k-2} X_{2}+\cdots+\tilde{E}_{k-M} X_{M} \\
& =O\left(k^{2}\right) \text { as } k \rightarrow \pm \infty .
\end{aligned}
$$

An approximate system of equations for the coefficients $X_{j}, j=1, \ldots, M$, in (18), and hence for the singularity locations for the function $u(x)$, results from (31), (32), and (33):

$$
\tilde{F}_{k}+\tilde{F}_{k-1} X_{1}+\tilde{F}_{k-2} X_{2}+\cdots+\tilde{F}_{k-M} X_{M}=0,
$$

where the requirement that the equations shall be independent normally means that we take $k=N / 2-M, \ldots, N / 2-1$, assuming that $N>8 M$. From (32) and (33) it is clear that (34) is an algebraic equation of degree 3 for the coefficients $X_{j}, j=1, \ldots, M$. From the estimates we have given above, we expect that the error in the solution of (34) for $X_{j}$ will be of the order $O\left(|N|^{-3}\right)$ as $N \rightarrow \infty$. This is one order better than the expected error in the solutions of (26) for the case where $Q=1$ considered in $\S 3.1$.

3.3. The general case. For arbitrary $Q$, the relations (21) can be written

$$
\sum_{n=0}^{Q}(i k)^{Q-n} B_{k}^{n}=(i k)^{Q} C_{k}, \quad k= \pm 1, \pm 2, \ldots
$$


We introduce the following notation for $k \neq 0,1,2, \ldots, M$ :

$$
\begin{gathered}
G_{k}^{Q}(1) \stackrel{\text { def }}{=} k^{Q} C_{k}+(k-1)^{Q} C_{k-1} X_{1}+(k-2)^{Q} C_{k-2} X_{2} \\
+\cdots+(k-M)^{Q} C_{k-M} X_{M},
\end{gathered}
$$

and then successively for $n=2,3, \ldots, Q+1$ and $k \neq 0,1,2, \ldots, n M$, define the quantities

$$
\begin{aligned}
G_{k}^{Q}(n) \stackrel{\text { def }}{=} & G_{k}^{Q}(n-1)+G_{k-1}^{Q}(n-1) X_{1}+G_{k-2}^{Q}(n-1) X_{2} \\
& +\cdots+G_{k-M}^{Q}(n-1) X_{M} .
\end{aligned}
$$

Utilizing (20), we find from (35), (36), and (37) in the same way as (24) and (31) were established for the cases $Q=1$ and $Q=2$, respectively, that the following equations hold:

$$
G_{k}^{Q}(Q+1)=0, \quad k \neq 0,1,2, \ldots,(Q+1) M .
$$

From (36) and (37) it is clear that (38) for each $k$ is an algebraic equation of degree $Q+1$ with respect to the coefficients $X_{j}, j=1, \ldots, M$, in (18), and hence for the singularity locations for the function $u(x)$. From (15), (36), and (37) it is furthermore clear that the quantities $G_{k}^{Q}(n)$ are approximately known from the Fourier coefficients $u(x)$. In fact, for $k \neq 0,1,2, \ldots, M$, we have

$$
\begin{gathered}
G_{k}^{Q}(1) \approx \tilde{G}_{k}^{Q}(1) \stackrel{\text { def }}{=} k^{Q} \tilde{C}_{k}+(k-1)^{Q} \tilde{C}_{k-1} X_{1}+(k-2)^{Q} \tilde{C}_{k-2} X_{2} \\
+\cdots+(k-M)^{Q} \tilde{C}_{k-M} X_{M}=O\left(k^{Q}\right) \text { as } k \rightarrow \pm \infty .
\end{gathered}
$$

For $n=2,3, \ldots, Q+1$ and for $k \neq 0,1,2, \ldots, n M$, we successively have

$$
\begin{gathered}
G_{k}^{Q}(n) \approx \tilde{G}_{k}^{Q}(n) \stackrel{\text { def }}{=} \tilde{G}_{k}^{Q}(n-1)+\tilde{G}_{k-1}^{Q}(n-1) X_{1}+\tilde{G}_{k-2}^{Q}(n-1) X_{2} \\
+\cdots+\tilde{G}_{k-M}^{Q}(n-1) X_{M}=O\left(k^{Q}\right) \text { as } k \rightarrow \pm \infty .
\end{gathered}
$$

The difference between the two sides in both (39) and (40) is seen to be of the order $O\left(|k|^{-1}\right)$ as $k \rightarrow \pm \infty$. An approximate system of equations for the coefficients $X_{j}, j=1, \ldots, M$, in (18) therefore results from (38), (39), and (40):

$$
\tilde{G}_{k}^{Q}(Q+1)=0,
$$

where again the requirement that the equations shall be independent normally means that we take $k=N / 2-M, \ldots, N / 2-1$, assuming that $N>$ $2(Q+2) M$. From (39) and (40) it is clear that (41) is an algebraic equation of degree $Q+1$ for the coefficients $X_{j}, j=1, \ldots, M$. From the estimates we have given above, we expect that the error in the solution of (41) for $X_{j}$ will be of the order $O\left(|N|^{-Q-1}\right)$ as $N \rightarrow \infty$.

Since (41) constitutes a system of algebraic equations of degree $Q+1$, we cannot expect it to have a uniquely determined solution in general, and numerically we would have to apply a nonlinear solver, which normally implies some iterative strategy. Depending on the actual application, there may be a number 
of different strategies for obtaining the relevant solution of (41). One possible strategy clearly is to use the solution obtained by the method described in [3] for the case $Q=0$ as a first approximation for the iterations, an approach which will be considered further in $\S 5$. Having solved (41) for the $(Q+1)$ storder accurate approximations of the coefficients $X_{j}, j=1, \ldots, M$, we then solve the associated approximate equation (18). As in [3], we may finally from the $M$ roots $z_{j}, j=1, \ldots, M$, of (18) calculate approximations for the $M$ discontinuity locations $\gamma_{j}, j=1, \ldots, M$, on the interval $[-\pi, \pi)$ by the equations

$$
e^{-i \gamma_{j}}=\frac{z_{j}}{\left|z_{j}\right|}, \quad j=1, \ldots, M .
$$

It seems reasonable to expect that the singularity locations calculated in this way are at least $(Q+1)$ st-order accurate also.

In the above approximate calculation of the singularity locations, the representation (9) is only used in order to obtain an error estimate and not in the actual calculation itself. If necessary, we are therefore free to use the equation (41) with $Q=Q+1$ in order to guarantee that the obtained approximate singularity locations are at least $(Q+2)$ st-order accurate. By solving the associated system (16), the reconstruction described in $\S 2$ will then lead to a $(Q+1)$ storder accurate representation of the function $u(x)$ in the form (9). As we shall see in the following sections, however, we are in most cases in the fortunate situation that numerical experiments indicate that the accuracy obtained by the above calculation of the singularity locations is much better than the pessimistic estimates we have given. A lower value of $Q$ than that used in the representation (9), may therefore often suffice in the calculation of the singularity locations by (18), (41), (42).

\section{AN EXAMPLE}

We shall in this section consider a $2 \pi$-periodic function $u(x)$ which is known to be piecewise smooth on $[-\pi, \pi]$ and discontinuous with one (and only one) discontinuity in each period. From the discussion in $\S 2$, we then know that the Fourier coefficients associated with the function $u(x)$ will asymptotically as $k \rightarrow \pm \infty$ satisfy

$$
\hat{u}_{k}=\frac{1}{2 \pi i k} A e^{-i k \gamma}+O\left(|k|^{-2}\right),
$$

where the constant $\gamma$ denotes the actual location of the discontinuity in $[-\pi, \pi)$ and the constant $A$ the associated jump of $u(x)$ at that point.

We would first like to consider in detail the method of reconstruction described in [3] for this function $u(x)$, and thereby pinpoint some of the limitations involved in the approach. Thus $M=1$, and consequently $z=e^{-i \gamma}$ is known from (18) to approximately satisfy

$$
z+X_{1}=0,
$$

where the coefficient $X_{1}$ is calculated by the procedure described in [3], which here actually means solving the linear algebraic equation obtained from (41) by 
putting $Q=0$ and $M=1$. Thus we have

$$
\tilde{C}_{N / 2-1}+\tilde{C}_{N / 2-2} X_{1}=0 \text {, }
$$

where $\tilde{C}_{k}$ is given by (15). As a result of the equations (15), (44), and (45) we get

$$
z=-X_{1}=\frac{\tilde{C}_{N / 2-1}}{\tilde{C}_{N / 2-2}}=\frac{(N / 2-1) \hat{u}_{N / 2-1}}{(N / 2-2) \hat{u}_{N / 2-2}},
$$

which can be easily calculated if the truncated Fourier series expansion (1) is known.

On the other hand, we see from (15) and (43) that

$$
\tilde{C}_{k}=2 \pi i k \hat{u}_{k}=A e^{-i k \gamma}+H_{k},
$$

where $H_{k}=O\left(|k|^{-1}\right)$ as $k \rightarrow \pm \infty$. Thus (46) also gives

$$
z=\frac{A e^{-i(N / 2-1) \gamma}+H_{N / 2-1}}{A e^{-i(N / 2-2) \gamma}+H_{N / 2-2}}=e^{-i \gamma}+\frac{1}{\tilde{C}_{N / 2-2}}\left(H_{N / 2-1}-e^{-i \gamma} H_{N / 2-2}\right) .
$$

Since $A \neq 0$ by assumption, it follows from (47) that $\tilde{C}_{k}=O(1)$ as $k \rightarrow$ $\pm \infty$. Consequently, (48) shows that in general we may expect that

$$
z=e^{-i \gamma}+O\left(N^{-1}\right) \text { as } N \rightarrow \infty,
$$

and therefore

$$
\gamma=-\arg (z)+O\left(N^{-1}\right) \text { as } N \rightarrow \infty .
$$

It thus appears that the approximate discontinuity location determined by the method described in [3] is only first-order accurate in general. Since better accuracy than this was observed for the calculations reported in [3], the structure of the function $u(x)$ considered must be of importance for the accuracy of the calculations. In order to get more insight into that problem, we consider the special case of (43) where for $k \neq 0$

$$
\hat{u}_{k}=\frac{1}{2 \pi i k} A e^{-i k \gamma}+\frac{1}{2 \pi(i k)^{2}} B e^{-i k \mu}+\hat{v}_{k},
$$

where $A, B, \gamma, \mu$ are given constants with $A \neq 0,-\pi \leq \gamma<\pi,-\pi \leq \mu<\pi$, and $\hat{v}_{k}=O\left(|k|^{-3}\right)$ as $k \rightarrow \pm \infty$. With (51), we know from the discussion in $\S 2$ that the function $u(x)$ is discontinuous with jump $A$ at the points $x=$ $\gamma+2 m \pi, m=0, \pm 1, \pm 2, \ldots$, while $u^{\prime}(x)$ is discontinuous with jump $B$ at the points $x=\mu+2 m \pi, m=0, \pm 1, \pm 2, \ldots$. From (47), (51) it is easily seen that as $k \rightarrow \pm \infty$, we have

$$
H_{k}-e^{-i \gamma} H_{k-1}=\frac{\left(e^{-i \mu}-e^{-i \gamma}\right) k-e^{-i \mu}}{i k(k-1)} B e^{-i(k-1) \mu}+O\left(|k|^{-2}\right) .
$$

When $B \neq 0$ we may therefore conclude from (48) that unless $\mu=\gamma$, the approximate discontinuity location $-\arg (z)$ determined by (46) will in fact only be first-order accurate. When $B=0$ or $\mu=\gamma$, however, (48) and (52) show 
that the approximate discontinuity location $-\arg (z)$ will be at least secondorder accurate. For a function $u(x)$ with only one discontinuity in each period, we have thus actually proved that a sufficient condition for second-order accuracy is that $u(x)$ is continuously differentiable at every point where $u(x)$ is continuous.

Based on this result and a large number of numerical experiments, we conjecture that a sufficient condition for the method of reconstruction of a discontinuous function $u(x)$ described in [3] to be accurate (i.e. the discontinuity locations determined with second-order accuracy, and the associated jumps with first-order accuracy) is that $u(x)$ is continuously differentiable everywhere except at the points where $u(x)$ is discontinuous. When the function $u(x)$ has more than one discontinuity in each period, the formal proof of this conjecture is open, but the numerical evidence is very convincing. We shall later formulate an extension of this conjecture to the more general reconstruction which we have described earlier in this paper.

If $A=0, B \neq 0$ in (51), it follows from the discussion in $\S 2$ that the underlying function $u(x)$ is continuous everywhere, but $u^{\prime}(x)$ is discontinuous with jump $B$ at the points $x=\mu+2 m \pi, m=0, \pm 1, \pm 2, \ldots$. The method described in [3] is not designed for such functions, but it is easy to see that the formula (46) in this case gives

$$
z=\frac{N-4}{N-2} e^{-i \mu}+O\left(N^{-p}\right) \text { as } N \rightarrow \infty,
$$

where $p \geq 1$. Thus, the singularity location is also in this case satisfying $\mu=-\arg (z)+O\left(N^{-1}\right)$ as $N \rightarrow \infty$. We note that if we assume that the function $u(x)$ is such that $u^{\prime}(x)$ is continuously differentiable everywhere except at the single point in each period where $u^{\prime}(x)$ is discontinuous, it can be shown by a calculation similar to that given above for (47)-(52), that $p \geq 2$ in (53).

In the case where $A \neq 0, B \neq 0$, and $\gamma \neq \mu$ in (51), we have seen above that with $M=1$ the method described in [3] for the determination of the discontinuity locations is only first-order accurate. Numerical experiments do show, however, that if in this case we apply the method described in [3] with $M=2$, we obtain improved accuracy. Although so far we have no theory supporting our conclusion, the two roots $z_{1}, z_{2}$ of equation (18) then seem to determine both singularity locations in each period by (42), where normally the point $x=\gamma$ is more accurately determined than the point $x=\mu$. In this connection it is interesting also to note that the asymptotic behavior we observe for $\left|z_{1}\right|$ and $\left|z_{2}\right|$ are different. In fact, for the root corresponding to the discontinuity in the function $u(x)$ itself we normally get $|z|=1+O\left(N^{-2}\right)$ as $N \rightarrow \infty$, while for the root corresponding to the discontinuity in $u^{\prime}(x)$ we normally get an asymptotic behavior corresponding to that seen in (53). In addition to determining approximate singularity locations, it therefore seems that the method described in [3] can also provide valuable information about the nature of these singularities. We hope to be able to follow up these observations in more detail elsewhere.

Further improvements in the determination of the singularity locations for the case where $A \neq 0, B \neq 0$ in (51) can be obtained by the method described in $\S 3.1$. If $\gamma \neq \mu$, it is then consistent to take $M=2$. If we restrict ourselves to 
the case where $\gamma=\mu$, however, it is consistent to take $M=1$, and $z=e^{-i \gamma}$ is still approximately determined by (44). Now, however, $X_{1}$ has to be calculated by the quadratic algebraic equation (26) which here reduces to

$$
(N / 2-1) \tilde{C}_{N / 2-1}+2(N / 2-2) \tilde{C}_{N / 2-2} X_{1}+(N / 2-3) \tilde{C}_{N / 2-3} X_{1}^{2}=0
$$

From (15) and (51) we have that

$$
k \tilde{C}_{k}=2 \pi i k^{2} \hat{u}_{k}=(k A-i B) e^{-i k \gamma}+W_{k},
$$

where $W_{k}=2 \pi i k^{2} \hat{v}_{k}=O\left(|k|^{-1}\right)$ as $k \rightarrow \pm \infty$. When $A \neq 0$, the two solutions $X_{1}^{(1)}, X_{1}^{(2)}$ of (54) must clearly satisfy

$$
\begin{aligned}
X_{1}^{(1)} X_{1}^{(2)} & =\frac{(N / 2-1) \tilde{C}_{N / 2-1}}{(N / 2-3) \tilde{C}_{N / 2-3}}=\frac{[(N / 2-1) A-i B] e^{-i(N / 2-1) \gamma}+W_{N / 2-1}}{[(N / 2-3) A-i B] e^{-i(N / 2-3) \gamma}+W_{N / 2-3}} \\
= & \frac{N-2}{N-6} e^{-2 i \gamma}+O\left(N^{-2}\right) \text { as } N \rightarrow \infty \\
X_{1}^{(1)}+X_{1}^{(2)} & =-2 \frac{(N / 2-2) \tilde{C}_{N / 2-2}}{(N / 2-3) \tilde{C}_{N / 2-3}} \\
& =-2 \frac{[(N / 2-2) A-i B] e^{-i(N / 2-2) \gamma}+W_{N / 2-2}}{[(N / 2-3) A-i B] e^{-i(N / 2-3) \gamma}+W_{N / 2-3}} \\
& =-2 \frac{N-4}{N-6} e^{-i \gamma}+O\left(N^{-2}\right) \text { as } N \rightarrow \infty .
\end{aligned}
$$

Thus, when $A \neq 0$, we may conclude that the two solutions of equation (54) will asymptotically be such that as $N \rightarrow \infty$

$$
X_{1}^{(1)}=-e^{-i \gamma}+O\left(N^{-2}\right), \quad X_{1}^{(2)}=-\frac{N-2}{N-6} e^{-i \gamma}+O\left(N^{-2}\right) .
$$

The solution which is at least second-order accurate is therefore $X_{1}^{(1)}$. If we assume that the function $u(x)$ is piecewise smooth on $[-\pi, \pi]$ and has one (and only one) singularity in each period, $u(x), u^{\prime}(x), u^{\prime \prime}(x)$ etc. are all necessarily continuously differentiable everywhere except at that singularity. By a calculation similar to the one given above for (47)-(52), it is not difficult to show that $X_{1}^{(1)}$ in this case actually has to be at least third-order accurate.

We observe from (58) that the other solution $X_{1}^{(2)}$ is a first-order accurate approximation for the exact value of $X_{1}$, and that the argument for the two solutions are equal to at least second-order. Thus, equation (42) would in this particular case have led to an approximate solution for the singularity location which is at least second-order accurate, even if we had picked the less accurate solution of (54).

When $A=0$, it is easily seen that the difference between the two roots of (54) will be of the order $O\left(|N|^{-2}\right)$ as $N \rightarrow \infty$. In that case there will therefore be no clear preference for either of the two roots of (54). However, this case corresponds to a case where the underlying function $u(x)$ is continuous everwhere, but with $u^{\prime}(x)$ discontinuous. Since the method we have described 
in this paper is not optimal for such functions, we shall restrict ourselves to giving some brief remarks in $\S 6$ on such cases.

\section{NUMERICAL CONSIDERATIONS}

Since the method described in [3] already provides us with an accurate approximation for the required solution of (41), a strategy for improving that approximation is to apply the well-known Newton-Raphson method [14]. For that purpose we need to calculate the Jacobi matrix associated with the system of equations (41). We shall therefore consider the structure of these equations in more detail. By introducing the notation

$$
H_{k}^{Q}(1)=(k-1)^{Q} \tilde{C}_{k-1} X_{1}+(k-2)^{Q} \tilde{C}_{k-2} X_{2}+\cdots+(k-M)^{Q} \tilde{C}_{k-M} X_{M},
$$

and then successively for $n=2,3, \ldots, Q+1$,

$$
H_{k}^{Q}(n)=H_{k-1}^{Q}(n-1) X_{1}+H_{k-2}^{Q}(n-1) X_{2}+\cdots+H_{k-M}^{Q}(n-1) X_{M}
$$

we see that as long as $k \neq 0,1,2, \ldots,(Q+1) M$, the quantity $H_{k}^{Q}(n)$ will for each $n=1,2,3, \ldots, Q+1$ be an algebraic expression, homogeneous of degree $n$ with respect to the coefficients $X_{1}, X_{2}, \ldots, X_{M}$, and from (39) it readily follows that

$$
\tilde{G}_{k}^{Q}(1)=k^{Q} \tilde{C}_{k}+H_{k}^{Q}(1) .
$$

By induction it is not difficult to see that for $n=2,3, \ldots, Q+1$ we have the following relations:

$$
\begin{aligned}
\tilde{G}_{k}^{Q}(n)= & k^{Q} \tilde{C}_{k}+\left(\begin{array}{c}
n \\
1
\end{array}\right) H_{k}^{Q}(1)+\left(\begin{array}{c}
n \\
2
\end{array}\right) H_{k}^{Q}(2) \\
& +\cdots+\left(\begin{array}{c}
n \\
n-1
\end{array}\right) H_{k}^{Q}(n-1)+H_{k}^{Q}(n),
\end{aligned}
$$

where $\left(\begin{array}{l}n \\ m\end{array}\right)$ denote the usual binomial coefficients.

As long as $k \neq 0,1,2, \ldots,(Q+1) M$, it is furthermore easy from the above relations to show by induction that for each $j=1,2, \ldots, M$ and for each $n=2,3, \ldots, Q+1$, we have

$$
\begin{aligned}
\frac{\partial}{\partial X_{j}} H_{k}^{Q}(n) & =n H_{k-j}^{Q}(n-1), \\
\frac{\partial}{\partial X_{j}} \tilde{G}_{k}^{Q}(n) & =n \tilde{G}_{k-j}^{Q}(n-1) .
\end{aligned}
$$

Thus the elements of the Jacobian matrix for the system of equations (41) are obtainable by straightforward algebraic calculations, making the NewtonRaphson method look even more attractive.

The implementation of the algorithms described in this paper have not encountered any difficulties. For the implementation of the Newton-Raphson method we have essentially followed the scheme given in [14]. Since we here are concerned with systems involving complex variables, however, we have utilized LINPACK subroutines [2] in order to solve the respective linear systems 
of algebraic equations. For the determination of the discontinuity locations, the roots of the relevant polynomial (18) have been calculated by the Laguerre method, where, as in [3], we have been able to use the subroutines provided in [14].

To illustrate the approach described, we consider three examples. The first is one of the examples studied in [3], namely the $2 \pi$-periodic extension of the following piecewise polynomial function of degree 2 with five discontinuities in each period:

$$
f(x)= \begin{cases}2+x-4 x^{2} & \text { if } 1.0<x<1.5 \\ -5+x+x^{2} & \text { if } 1.5<x<2.5 \\ 2-3 x+2 x^{2} & \text { if } 2.5<x<3.5 \\ 1-2 x+x^{2} & \text { if } 3.5<x<5.0 \\ 40+x-x^{2} & \text { if } 5.0<x<2 \pi+1.0\end{cases}
$$

The absolute value of the largest error in the calculated discontinuity locations for the function (65) for different values of $N$ and $Q$ were found to be

\begin{tabular}{|c|c|c|c|c|}
\hline$N$ & 64 & 128 & 256 & 512 \\
\hline \hline $\mathrm{Q}=0$ & $0.58 \cdot 10^{-2}$ & $0.12 \cdot 10^{-2}$ & $0.32 \cdot 10^{-3}$ & $0.68 \cdot 10^{-4}$ \\
$\mathrm{Q}=1$ & $0.80 \cdot 10^{-3}$ & $0.11 \cdot 10^{-4}$ & $0.10 \cdot 10^{-5}$ & $0.48 \cdot 10^{-7}$ \\
$\mathrm{Q}=2$ & $0.25 \cdot 10^{-10}$ & $0.19 \cdot 10^{-9}$ & $0.59 \cdot 10^{-8}$ & $0.37 \cdot 10^{-7}$ \\
$\mathrm{Q}=3$ & $0.34 \cdot 10^{-9}$ & $0.21 \cdot 10^{-7}$ & $0.12 \cdot 10^{-5}$ & $0.58 \cdot 10^{-2}$ \\
\hline \hline $2 \pi / N$ & 0.0982 & 0.0491 & 0.0245 & 0.0123 \\
\hline
\end{tabular}

Secondly, we consider the $2 \pi$-periodic extension of the following piecewise polynomial function of degree 3 with four discontinuities in each period:

$$
f(x)= \begin{cases}0 & \text { if } 0<x<1.0 \\ 3+x+2 x^{2}-3 x^{3} & \text { if } 1.0<x<2.0 \\ 2+4 x-5 x^{2}+x^{3} & \text { if } 2.0<x<3.0 \\ 1-3 x+4 x^{2}-x^{3} & \text { if } 3.0<x<4.0 \\ 0 & \text { if } 4.0<x<2 \pi\end{cases}
$$

The absolute value of the largest error in the calculated discontinuity locations for the function (66) for different values of $N$ and $Q$ were found to be

\begin{tabular}{|c|c|c|c|c|}
\hline$N$ & 64 & 128 & 256 & 512 \\
\hline \hline $\mathrm{Q}=0$ & $0.42 \cdot 10^{-2}$ & $0.76 \cdot 10^{-3}$ & $0.16 \cdot 10^{-3}$ & $0.40 \cdot 10^{-4}$ \\
$\mathrm{Q}=1$ & $0.83 \cdot 10^{-4}$ & $0.55 \cdot 10^{-5}$ & $0.14 \cdot 10^{-6}$ & $0.12 \cdot 10^{-7}$ \\
$\mathrm{Q}=2$ & $0.16 \cdot 10^{-4}$ & $0.59 \cdot 10^{-6}$ & $0.29 \cdot 10^{-7}$ & $0.31 \cdot 10^{-8}$ \\
$\mathrm{Q}=3$ & $0.98 \cdot 10^{-9}$ & $0.37 \cdot 10^{-8}$ & $0.92 \cdot 10^{-7}$ & $0.13 \cdot 10^{-2}$ \\
$\mathrm{Q}=4$ & $0.20 \cdot 10^{-7}$ & $0.48 \cdot 10^{-6}$ & 2.14 & 0.77 \\
\hline
\end{tabular}


As a final example, we consider the $2 \pi$-periodic extension of the following piecewise smooth function with three discontinuities in each period:

$$
f(x)= \begin{cases}0 & \text { if } 0<x<1.0 \\ \mathrm{e}^{x} & \text { if } 1.0<x<2.0 \\ \cos \frac{x}{2} & \text { if } 2.0<x<5.0 \\ 0 & \text { if } 5.0<x<2 \pi\end{cases}
$$

The absolute value of the largest error in the calculated discontinuity locations for the function (67) for different values of $N$ and $Q$ were found to be

\begin{tabular}{|c|c|c|c|c|c|}
\hline$N$ & 32 & 64 & 128 & 256 & 512 \\
\hline \hline $\mathrm{Q}=0$ & $0.78 \cdot 10^{-2}$ & $0.14 \cdot 10^{-2}$ & $0.31 \cdot 10^{-3}$ & $0.73 \cdot 10^{-4}$ & $0.18 \cdot 10^{-4}$ \\
$\mathrm{Q}=1$ & $0.73 \cdot 10^{-3}$ & $0.22 \cdot 10^{-4}$ & $0.10 \cdot 10^{-5}$ & $0.56 \cdot 10^{-7}$ & $0.27 \cdot 10^{-8}$ \\
$\mathrm{Q}=2$ & $0.11 \cdot 10^{-3}$ & $0.40 \cdot 10^{-5}$ & $0.12 \cdot 10^{-6}$ & $0.51 \cdot 10^{-8}$ & $0.27 \cdot 10^{-9}$ \\
$\mathrm{Q}=3$ & $0.97 \cdot 10^{-4}$ & $0.12 \cdot 10^{-6}$ & $0.28 \cdot 10^{-9}$ & $0.53 \cdot 10^{-10}$ & $0.44 \cdot 10^{-4}$ \\
$\mathrm{Q}=4$ & - & $0.36 \cdot 10^{-8}$ & $0.13 \cdot 10^{-9}$ & 1.1 & 0.70 \\
$\mathrm{Q}=5$ & - & $0.42 \cdot 10^{-9}$ & 0.86 & 0.81 & 0.52 \\
\hline
\end{tabular}

From the above numerical results we can conclude that when, as in our numerical calculations, double precision on a PC ( 53 bits) is employed, the robustness of the described approach deteriorates when $Q>3$. The reason for this is simply that the number of solutions of the considered system of equations (41) increases with $Q$ since (41) is an algebraic system of degree $Q+1$ with respect to the unknown quantities $X_{1}, X_{2}, \ldots, X_{M}$, and consequently the system (41) becomes more and more ill-conditioned as $Q$ grows.

For the first two examples we see that when $Q=3$, the most accurate results are obtained for the lowest values of $N$, and that the accuracy decreases with increasing $N$. The reason for this is twofold. On the one hand, the applied algorithm should according to the given theory be exact in those two cases for $Q=3$ when roundoff errors are disregarded. On the other hand, the example considered in the preceding section shows that the different solutions of the system (41) can be expected to be more and more clustered with increasing $N$. Thus it should be expected that the system (41) becomes more and more ill-conditioned as $N$ grows.

The numerical results obtained for the first example (65) also confirm the above conclusions. The reason why $Q=2$ in this case gives the most accurate results is simply that according to the given theory, the algorithm applied should in that case be exact for every $Q \geq 2$ when roundoff errors are disregarded.

For functions which at the discontinuity locations have jumps also in the derivatives of all orders, such as the function (67), we would again like to stress that the numerical experiments do indicate that in most cases the accuracy obtained by the above calculation of the singularity locations is at least one order better than the pessimistic estimates we have given in the earlier sections. In fact, we conjecture that if the function $u(x)$ is $Q+1$ times continuously differentiable everywhere on each of the intervals between the detected singularity locations, the approximations for the singularity locations for $u(x)$ will be determined with at least $(Q+2)$ nd-order accuracy from $(41)$ without having to 
put $Q:=Q+1$. This is the conjecture mentioned in the preceding section, which generalizes the property proved for the special case considered there. For the function (67), in particular, we see from the results given in the above table that asymptotically as $N \rightarrow \infty$, the error appears to be $O\left(N^{-2}\right)$ when $Q=0, O\left(N^{-4}\right)$ when $Q=1, O\left(N^{-4}\right)$ when $Q=2$, and amazingly $O\left(N^{-8}\right)$ when $Q=3$ for the smallest values of $N$ given (i.e., as long as the system (41) is sufficiently well-behaved). Usually (but not always), (41) therefore for $Q=1,2,3$ leads to sufficiently accurate approximations for the singularity locations, so that it may be advantageous to use a higher value of $Q$ in (9) than that employed in the solution of (41). This point will be further discussed elsewhere.

The numerical results given above are obtained by a numerical scheme which follows exactly the algorithms discussed. This implies that only Fourier coefficients $\hat{f}_{k}$ with $k>0$ are employed in the calculation of the discontinuity locations. For the function (67) this in particular means that when $N=32$ and $Q=3$, all 15 available Fourier coefficients $\hat{f}_{k}$ with $k>0$ are employed. Keeping this in mind, we feel that the accuracy obtained is particularly impressive, and that even more accurate results can be obtained if the scheme is modified to also take into consideration the available Fourier coefficients $\hat{f}_{k}$ with $k<0$. Some further remarks on how this can be done will be given in the following section.

The cost of applying the improved scheme discussed in this paper for the determination of the discontinuity locations is clearly higher than for the original scheme discussed in [3]. In fact, during each Newton-Raphson iteration an additional $M \times M$ linear system of algebraic equations has to be set up and solved. In order to ensure that the numerical scheme is sufficiently robust, we have in the calculations reported above successively improved the approximations of the desired solution of (41) by successively solving (41) for $Q=0,1,2, \ldots$ up to the desired value of $Q$, employing five Newton-Raphson iterations at each step except at $Q=0$. The numerical results given above are therefore obtained with a total number of $5 Q$ iterations, but numerical experiments clearly indicate that fewer iterations will normally be sufficient in order to obtain the indicated order of accuracy. The extra cost in applying the improved scheme for functions with a moderate or small number $M$ of discontinuities in each period will in practice be very small (fractions of a second on a PC).

As already indicated, the system of equations (41) to be solved becomes more and more ill-conditioned when $Q$ and/or $N$ grows. If higher accuracy than that obtained in the above calculations is desirable, the routines for solving the linear systems involved in the Newton-Raphson iterations should therefore be redesigned. We have so far not studied these ill-conditioned systems in detail, but an apparent strategy could be to apply the singular value decomposition method [5, p. 257]. Utilization of a higher precision at this stage in the calculations may perhaps also be an alternative. Since the systems in practice are small, these measures will not involve an excessively high cost, but will presumably make the calculations more feasible, and thus more accurate, for higher values of $Q$ as well as for higher values of $N$ than those shown in the above tables. 


\section{Discussion}

If we want to obtain an optimally accurate reconstruction, the number of terms known in the truncated Fourier series expansion, $N-1$, soon becomes the primary limiting factor. In particular, if the number of singularity locations in each period, $M$, is relatively large and/or we want to have the integer $Q$ relatively large in the reconstruction (9) of the function $u(x)$, limitations and inaccuracies may appear unless $N$ is chosen large. On the other hand, when $N$ is chosen large, we know that round-off errors in practice will severely limit the accuracy of the known highest-order Fourier coefficients as well as make the numerical scheme discussed in this paper more ill-conditioned. Thus the accuracy of the calculated reconstruction may be severely reduced when $N$ is large. Since only Fourier coefficients $\hat{u}_{k}$ with $k>0$ are utilized in the described reconstruction, however, the limitations and inaccuracies may be more severe than strictly necessary. By also utilizing the Fourier coefficients $\hat{u}_{k}$ with $k<0$, the accuracy of the reconstruction can be improved and the limitation $N>$ $2(Q+2) M$ in connection with (41), and $N>2(Q+1) M$ in connection with (16) can be relaxed. The other side of the coin here is, however, that all the equations resulting from (41) are then not necessarily independent in general. As briefly discussed in [3] for the special case $Q=0$, however, the least squares method [5, Chapter 5] for a properly weighted overdetermined system may then provide a viable alternative.

In the described reconstruction of functions of finite regularity, the number of singularity locations in each period, $M$, has been assumed to be known. For some applications, however, $M$ may not be known a priori, and would therefore have to be determined as part of the reconstruction. For such cases the described algorithms give us several options for actually determining the correct number $M$. In fact, consistent with the experience reported in $\S 4$, it normally suffices to consider the method described in [3], even if some of the singularities are associated with discontinuities in the derivatives of the function $u(x)$ and not in the function $u(x)$ itself. Thus, the brief discussion given in $[3, \S 6]$ for the special case $Q=0$ is relevant also for the general problem considered in this paper.

The general procedure which we so far have found most robust for determining the correct number of singularity locations in each period, say, $M_{1}$, is to choose a sufficiently large trial number, $\tilde{M}$, ensuring that $\tilde{M} \geq M_{1}$. We then look at the algorithm described in [3] for determining the coefficients in the corresponding algebraic equation (18) of degree $\tilde{M}$ for the locations of $\tilde{M}$ discontinuities in each period. That algorithm actually amounts to solving a system of linear algebraic equations obtained from (41) by putting $Q=0$ and $M=\tilde{M}$. If $\tilde{M} \geq M_{1}$, it is not difficult to see that the rank of the corresponding matrix in the exact linear system obtained from (38) by putting $Q=0$ and $M=\tilde{M}$ must be $M_{1}$. Thus, by calculating the numerical rank of the matrix for the approximate system (41) and eliminating near rank deficiencies, we do in fact obtain the correct number of singularities in each period, $M_{1}$. The singular value decomposition method seems to be a robust method for this purpose [5, Chapter 5].

We note that the design of the most efficient algorithm for the determination 
of the correct number of singularities will of course depend on what additional information can be inferred from the actual application considered. In this connection we would like to add that when we have been able to calculate the roots $z_{1}, \ldots, z_{\tilde{M}}$ of the algebraic equation (18) for a trial number $\tilde{M}$, numerical experiments indicate that on the one hand, these roots may in addition to information about the possible locations of the singularities also contain important information on the nature of these singularities, as was briefly discussed in $\S 4$. On the other hand, the roots obtained allow an immediate test of the correctness of the presumed number of singularities $\tilde{M}$, since the modulus of each of the obtained roots $\left|z_{1}\right|, \ldots,\left|z_{\tilde{M}}\right|$ should be not too far away from 1 . We hope to be able to follow up these considerations further elsewhere.

By letting the integer $Q$ increase with $N$, we are in principle always able to obtain reconstructions which are spectrally accurate as $N \rightarrow \infty$ by the procedure described in this paper. In practice, however, owing to the ill-conditioned equations that must be solved, the numerical experiments discussed in the preceding section show that we would have to restrict ourselves to some finite integer $Q$ which is relatively small, and $N$ should not be chosen too large either. The accuracy and the rate of convergence for the described reconstruction of the function $u(x)$ of finite regularity is therefore in practice primarily dependent on the smoothness of the remainder function $v(x)$ in the representation (9) for the function $u(x)$. That remainder function $v(x)$ is necessarily piecewise smooth in each period and $Q$ times continuously differentiable everywhere by the assumptions made, but not necessarily more regular than that in general. If the remainder function $v(x)$ for some finite integer $Q$ is infinitely smooth everywhere, the reconstruction will for that integer $Q$ in principle be spectrally accurate as $N \rightarrow \infty$. In particular, this means that the singularity locations are determined with spectral accuracy in that case. An alternative way of putting this result is that the described reconstruction will in principle be spectrally accurate as $N \rightarrow \infty$ if, for some fixed, finite integer $Q$, we restrict ourselves to functions $u(x)$ which are such that $u^{(q)}(x)$ does not have jumps at the singularity locations for any $q \geq Q+1$.

The ideas we have presented in this paper can be directly applied also to the problem of reconstruction of a piecewise smooth discontinuous function on the interval $[-1,1]$ from a known truncated Chebyshev series expansion. The most obvious approach is simply to transform the given Chebyshev series to a Fourier cosine series and use the methods described in this paper on the latter. Since the construction is completely analogous to that described in $[3, \S 4]$, we shall not discuss it further here.

We finally remark that the method of reconstruction of $2 \pi$-periodic functions of finite regularity $u(x)$ outlined in this paper is primarily described for discontinuous functions $u(x)$. In particular, the given error estimates presupposes that the function $u(x)$ is discontinuous. If the function $u(x)$ is continuous everywhere, but has discontinuities in some of its derivatives, the method and the accompanying error estimates could be improved by relatively minor modifications. In the equation (16) for instance, it is clearly not necessary in these cases to keep the terms representing discontinuities in the function $u(x)$ and those of the derivatives of $u(x)$ which are known to be continuous. The equation (41) for the determination of the coefficients in the equation for the singularity loca- 
tions (18) can also be made slightly better by a suitable modification of the given approach. Since the necessary modifications are relatively straightforward, we shall leave the details to the reader. We only note that such modified methods possibly may be of some value in connection with spline interpolations [13].

\section{ACKNOWLEDGMENT}

The author would like to thank Mr. Jens H. Rolfsnes for his assistance with the numerical computations reported in this paper.

\section{BIBLIOGRAPHY}

1. C. Canuto, M. Y. Hussaini, A. Quarteroni, and T. A. Zang, Spectral methods in fluid dynamics, Springer-Verlag, New York, 1988.

2. J. J. Dongarra, J. R. Bunch, C. B. Moler, and G. W. Stewart, LINPACK user's guide, SIAM, Philadelphia, PA, 1979.

3. K. S. Eckhoff, Accurate and efficient reconstruction of discontinuous functions from truncated series expansions, Math. Comp. 61 (1993), 745-763.

4. A. Erdélyi, W. Magnus, F. Oberhettinger, and F. C. Tricomi, Higher transcendental functions, McGraw-Hill, New York, 1953.

5. G. H. Golub and C. F. Van Loan, Matrix computations, 2nd ed., Johns Hopkins Univ. Press, Baltimore, MD, 1989.

6. D. Gottlieb, Issues in the application of high order schemes, Proc. Workshop on Algorithmic Trends in Computational Fluid Dynamics (Hampton, Virginia, U.S.A.), (M. Y. Hussaini, A. Kumar, and M. D. Salas, eds.), Springer-Verlag, ICASE/NASA LaRC Series, 1991, pp. 195-218.

7. D. Gottlieb, L. Lustman, and S. A. Orszag, Spectral calculations of one-dimensional inviscid compressible flows, SIAM J. Sci. Statist. Comput. 2 (1981), 296-310.

8. D. Gottlieb and S. A. Orszag, Numerical analysis of spectral methods: Theory and applications, SIAM, Philadelphia, PA, 1977.

9. F. B. Hildebrand, Introduction to numerical analysis, 2nd ed., Tata McGraw-Hill, New Delhi, 1974.

10. P. D. Lax, Accuracy and resolution in the computation of solutions of linear and nonlinear equations, Recent Advances in Numerical Analysis, Proc. Sympos. Univ. of WisconsinMadison (C. de Boor and G. H. Golub, eds.), Academic Press, New York, 1978, pp. 107117.

11. J. N. Lyness, The calculation of trigonometric Fourier coefficients, J. Comput. Phys. 54 (1984), 57-73.

12. G. Majda, W. A. Strauss, and M. Wei, Computation of exponentials in transient data, IEEE Trans. Antennas and Propagation 37 (1989), pp. 1284-1290.

13. M. J. D. Powell, Approximation theory and methods, Cambridge Univ. Press, Cambridge, 1981.

14. W. H. Press, B. P. Flannery, S. A. Teukolsky, and W. T. Vetterling, NUMERICAL RECIPES, the art of scientific computing, Cambridge Univ. Press, Cambridge, 1989.

15. R. Prony, Essai experimental et analytic ... , J. L'Ecole Polytech. (Paris), vol. 1, cahier 2, 1795, pp. 24-76.

16. A. Zygmund, Trigonometric series, Vol. I, Cambridge Univ. Press, Cambridge, 1968.

Department of Mathematics, University of Bergen, Johs. Brunsgt. 12, N-5008 Bergen, NORWAY

E-mail address: reske@mi.uib.no 